Supporting Information

\title{
Immobilization of Anions on Polymer Matrices for Gel Electrolytes with High Conductivity and Stability in Lithium Ion Batteries
}

\author{
Shih-Hong Wang, ${ }^{\dagger}$ Yong-Yi Lin, ${ }^{\dagger}$ Chiao-Yi Teng, ${ }^{\dagger}$ Yen-Ming Chen, ${ }^{\dagger}$ Ping-Lin Kuo, ${ }^{\dagger}$ \\ Yuh-Lang Lee, ${ }^{\dagger}$ Chien-Te Hsieh, ${ }^{*,+}$ and Hsisheng Teng $*, \dagger, \S$ \\ ${ }^{\dagger}$ Department of Chemical Engineering and Research Center for Energy Technology \\ and Strategy, National Cheng Kung University, Tainan 70101, Taiwan \\ ${ }^{ \pm}$Department of Chemical Engineering and Materials Science, Yuan Ze Fuel Cell \\ Center, Yuan Ze University, Taoyuan 32023, Taiwan \\ ${ }^{\S}$ Center for Micro/Nano Science and Technology, National Cheng Kung University, \\ Tainan 70101, Taiwan
}

*To whom correspondence should be addressed.

Hsisheng Teng: (E-mail): hteng@mail.ncku.edu.tw; (Fax): 886-6-2344496; (Tel): 886-6-2385371

Chien-Te Hsieh: (E-mail): cthsieh@saturn.yzu.edu.tw 
Supporting information for:

1. SEM image of PAV fibers and TEM image of PAVM:TiO fibers $_{2}$

2. Impedance data of electrolytes at various temperatures.

3. Linear scan $\left(0.2 \mathrm{mV} \mathrm{s}^{-1}\right)$ voltammograms

4. Charge-discharge profiles and cycle lifespans of batteries assembled with $\mathrm{LiNi}_{0.5} \mathrm{Mn}_{1.5} \mathrm{O}_{4}$.

5. SEM images of graphite anodes before and after charge-discharge cycling

6. C 1s XPS spectra of SEI layers.

7. Discharge capacity of graphite|electrolyte| $\mid \mathrm{LiFPO}_{4}$ batteries at various C-rates. 
1. SEM image of PAV fibers and TEM image of PAVM:TiO fibers $_{2}$

(a)

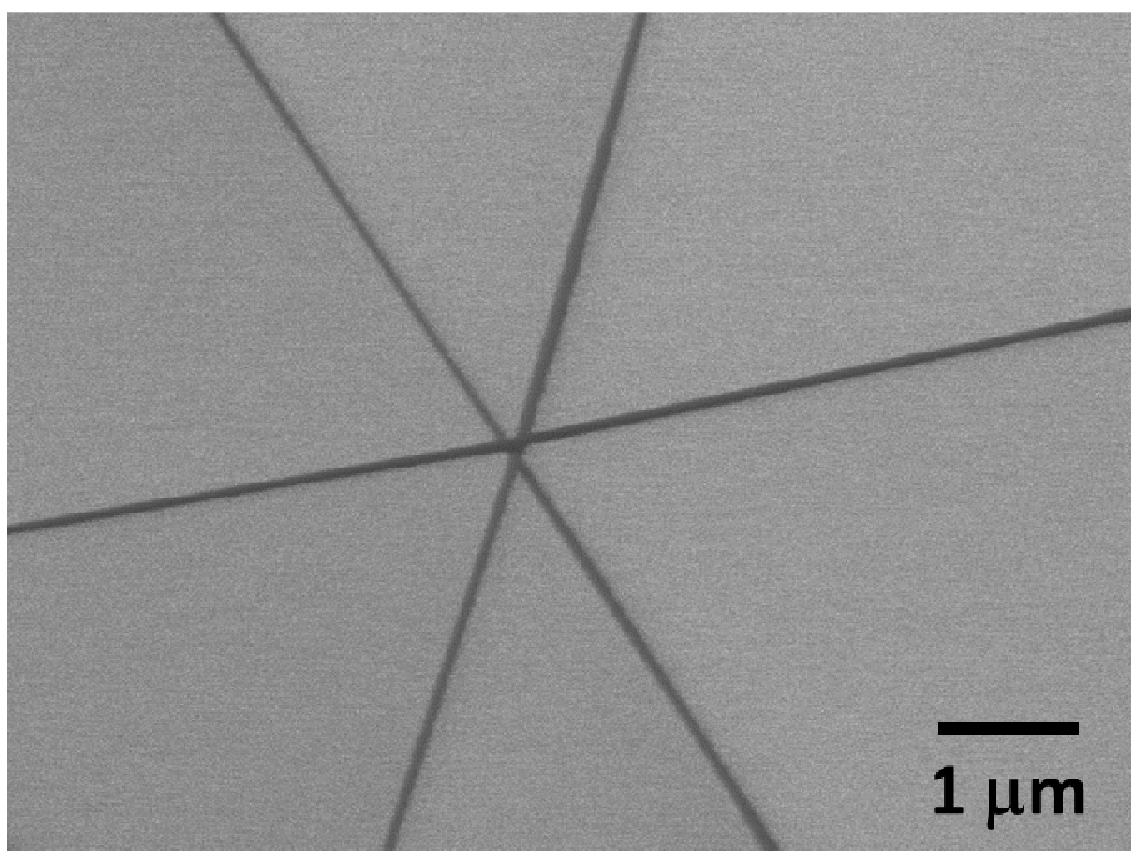

(b)

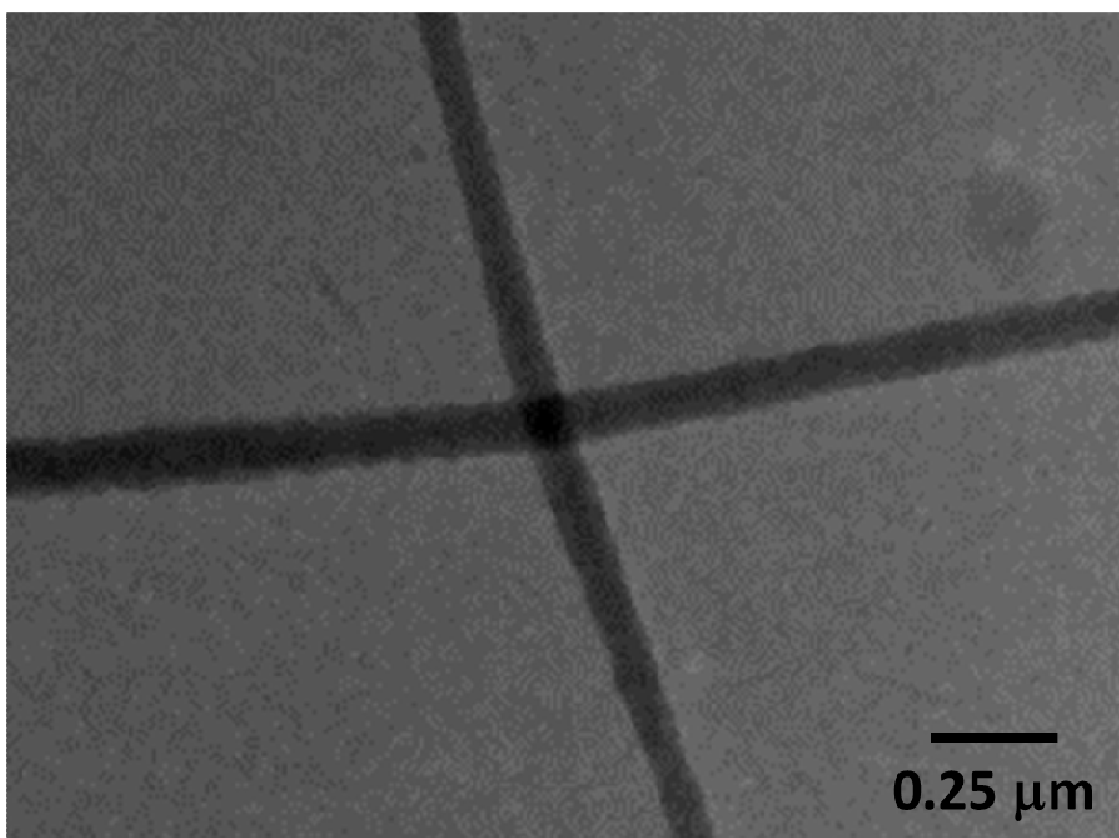

Figure S1. (a) SEM image of PAV fibers. (b) TEM image of $\mathrm{PAVM}: \mathrm{TiO}_{2}$ fibers. 
2. Impedance data of electrolytes at various temperatures
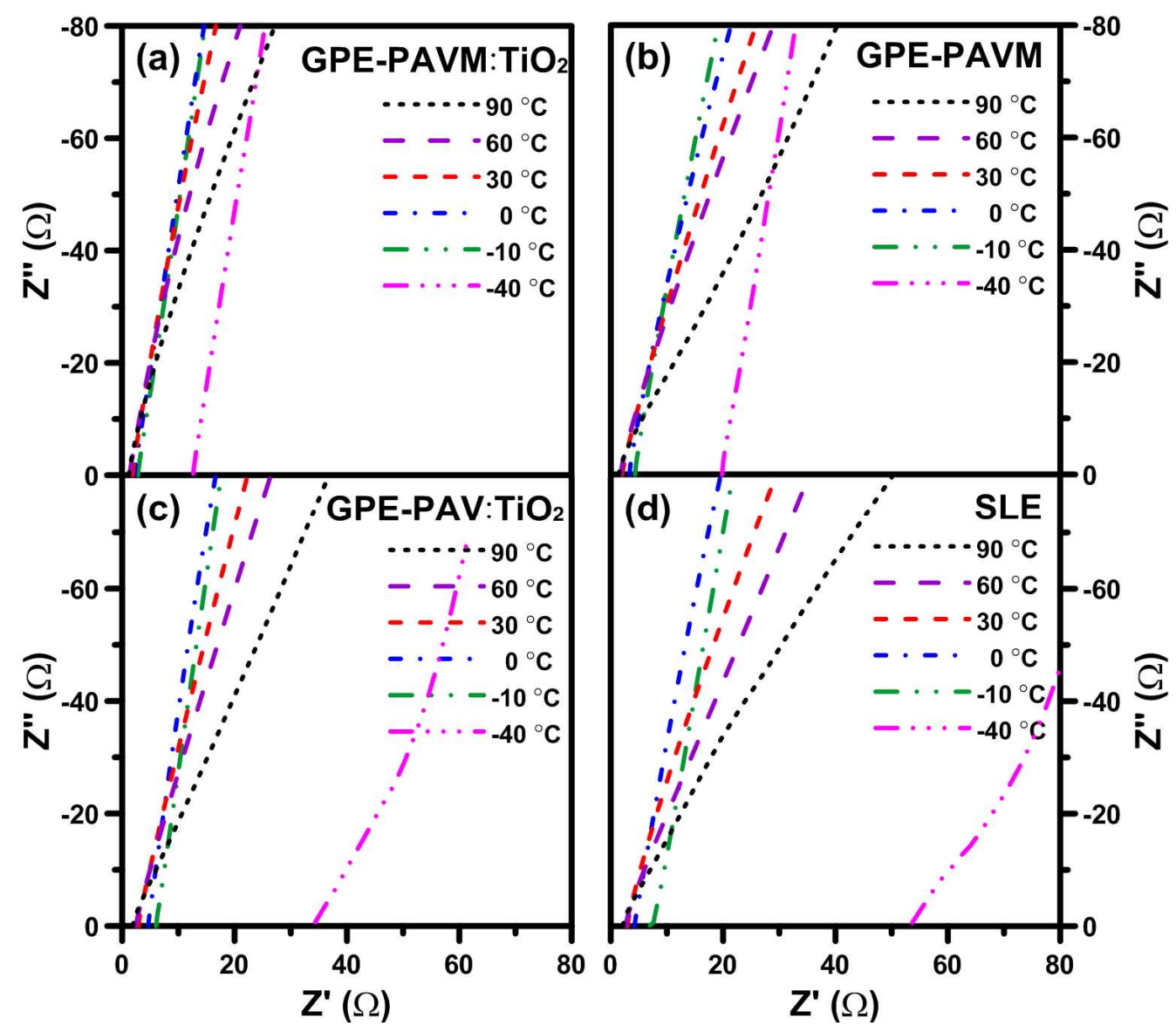

Figure S2. Nyquist impedance spectra of electrolytes at various temperatures: (a)

GPE-PAVM:TiO 2 ; (b) GPE-PAVM; (c) GPE-PAV:TiO 2 ; (d) SLE. The measurements were conducted by inserting the electrolytes between two stainless-steel electrodes and applying an $\mathrm{AC}$ potential amplitude of $5 \mathrm{mV}$ over a frequency range of $0.1 \mathrm{~Hz}$ to $100 \mathrm{k} \mathrm{Hz}$ at temperatures of -40 to $90{ }^{\circ} \mathrm{C}$. The thicknesses of the GPEs and SLE were 50 and $12 \mu \mathrm{m}$, respectively. 
3. Linear scan $\left(0.2 \mathrm{mV} \mathrm{s}^{-1}\right)$ voltammograms

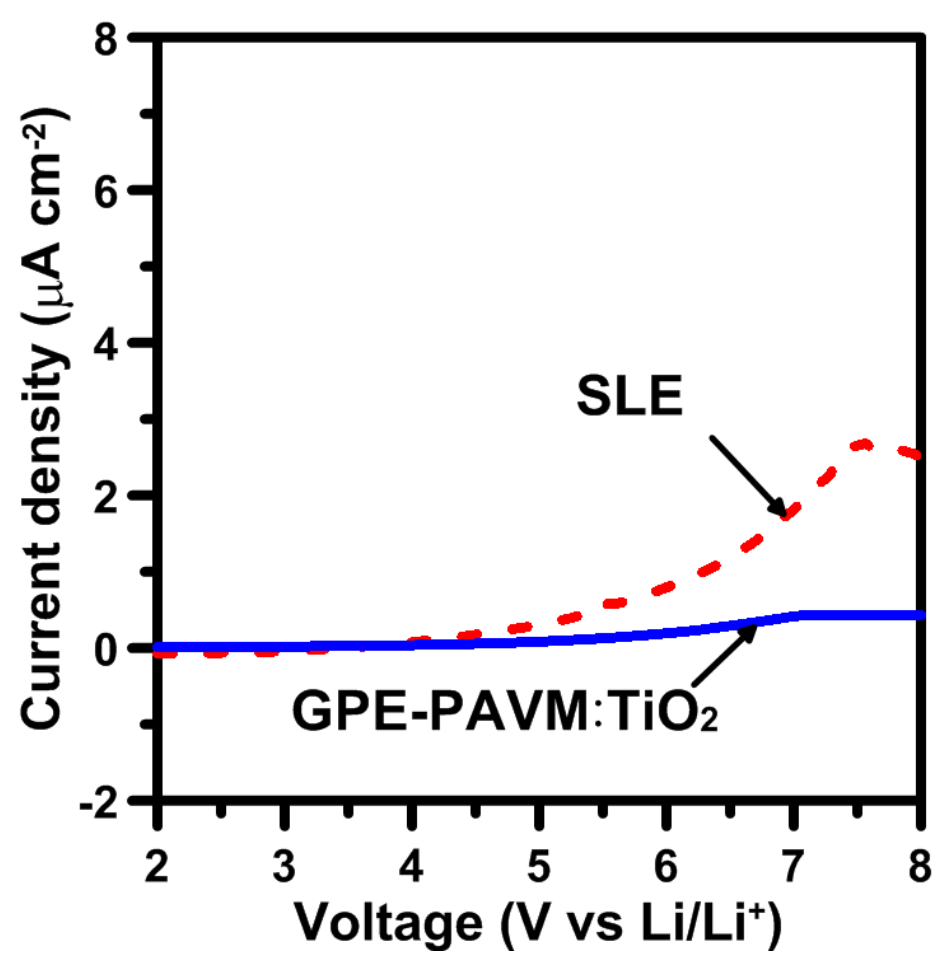

Figure S3. Linear scan $\left(0.2 \mathrm{mV} \mathrm{s}^{-1}\right)$ voltammograms of cells assembled by inserting an electrolyte (SLE or GPE-PAV: $\mathrm{TiO}_{2}$ ) between a working stainless-steel electrode and a Li-metal counter electrode. 
4. Charge-discharge profiles and cycle lifespans of batteries assembled with $\mathrm{LiNi}_{0.5} \mathrm{Mn}_{1.5} \mathrm{O}_{4}$
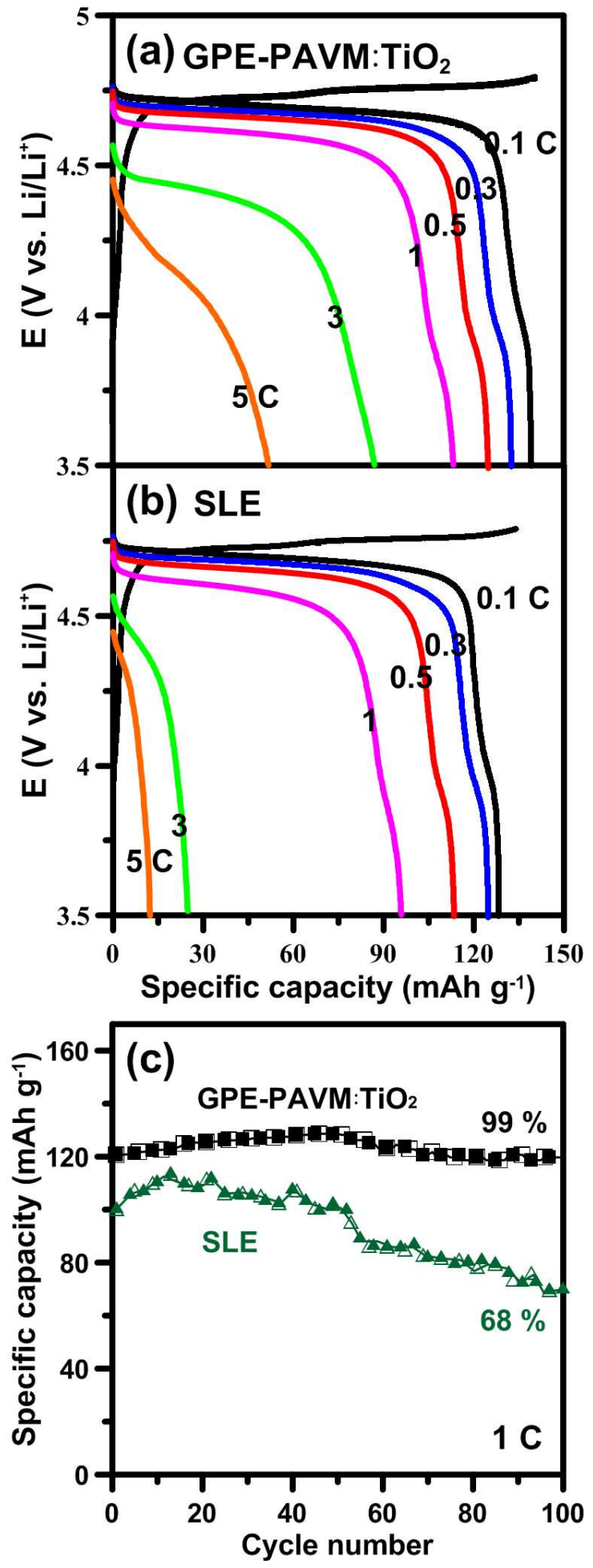
Figure S4. (a, b) Galvanostatic charge-discharge profiles of half-cell $\mathrm{LiNi}_{0.5} \mathrm{Mn}_{1.5} \mathrm{O}_{4} \mid$ electrolyte $\mid \mathrm{Li}$ batteries charged at $0.1 \mathrm{C}$-rate and discharged at various C-rates between 3.5 and 4.8 V: GPE-PAVM:TiO 2 (panel a); SLE (panel b). (c) Charge (solid symbols) and discharge (hollow symbols) capacities of the GPE-PAVM:TiO and SLE batteries following 100 cycles at $1 \mathrm{C}$. 
5. SEM images of graphite anodes before and after charge-discharge cycling

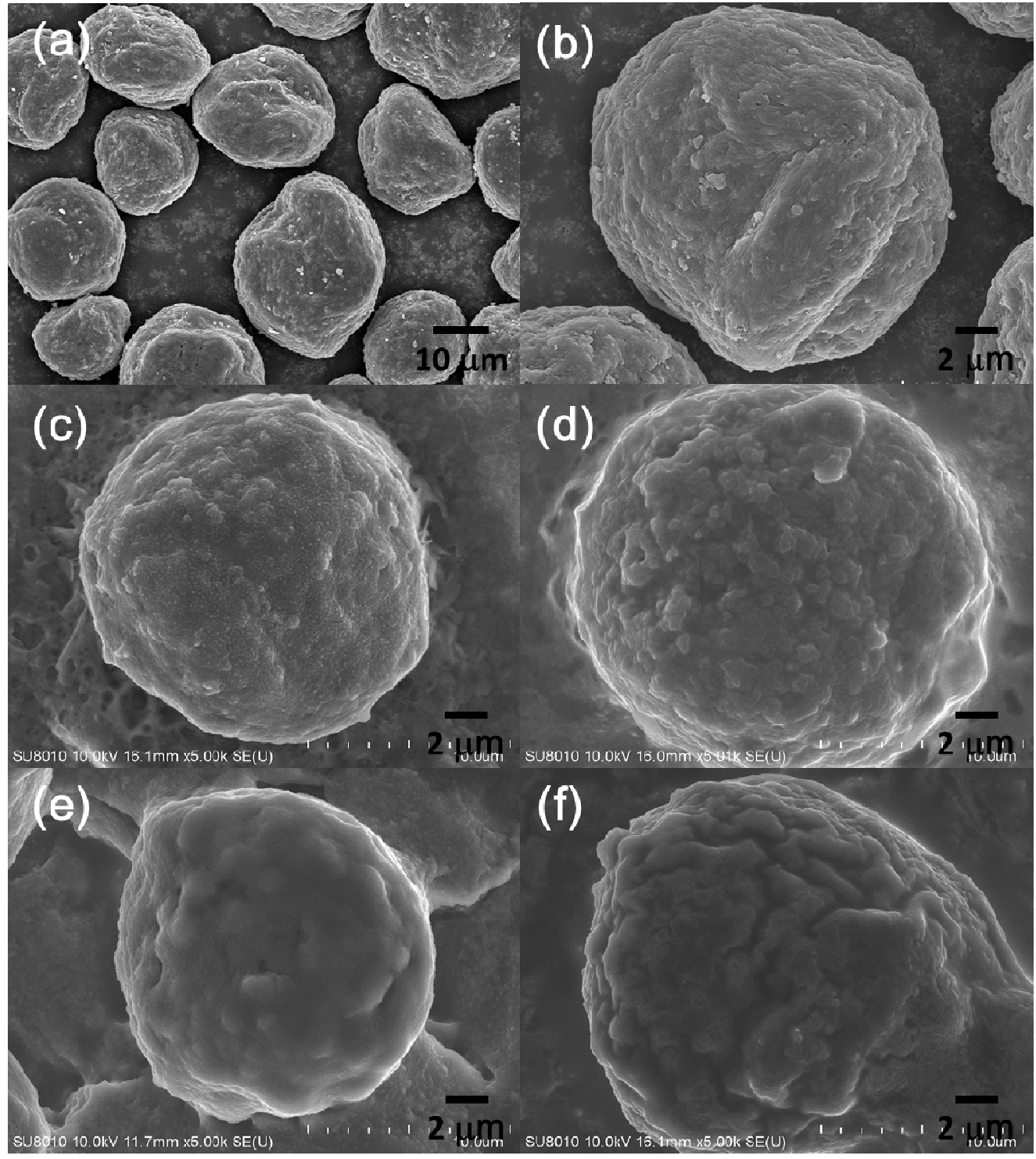

Figure S5. SEM images of pristine graphite powders before charge-discharge cycles $(a, b)$, and graphite powders of anodes in LIBs assembled using various electrolytes and subjected to a series of charge-discharge cycles: (c)

GPE-PAVM:TiO ${ }_{2}$; (d) GPE-PAVM; (e) GPE-PAV:TiO ${ }_{2}$; (f) SLE. 
6. C 1s XPS spectra of SEI layers
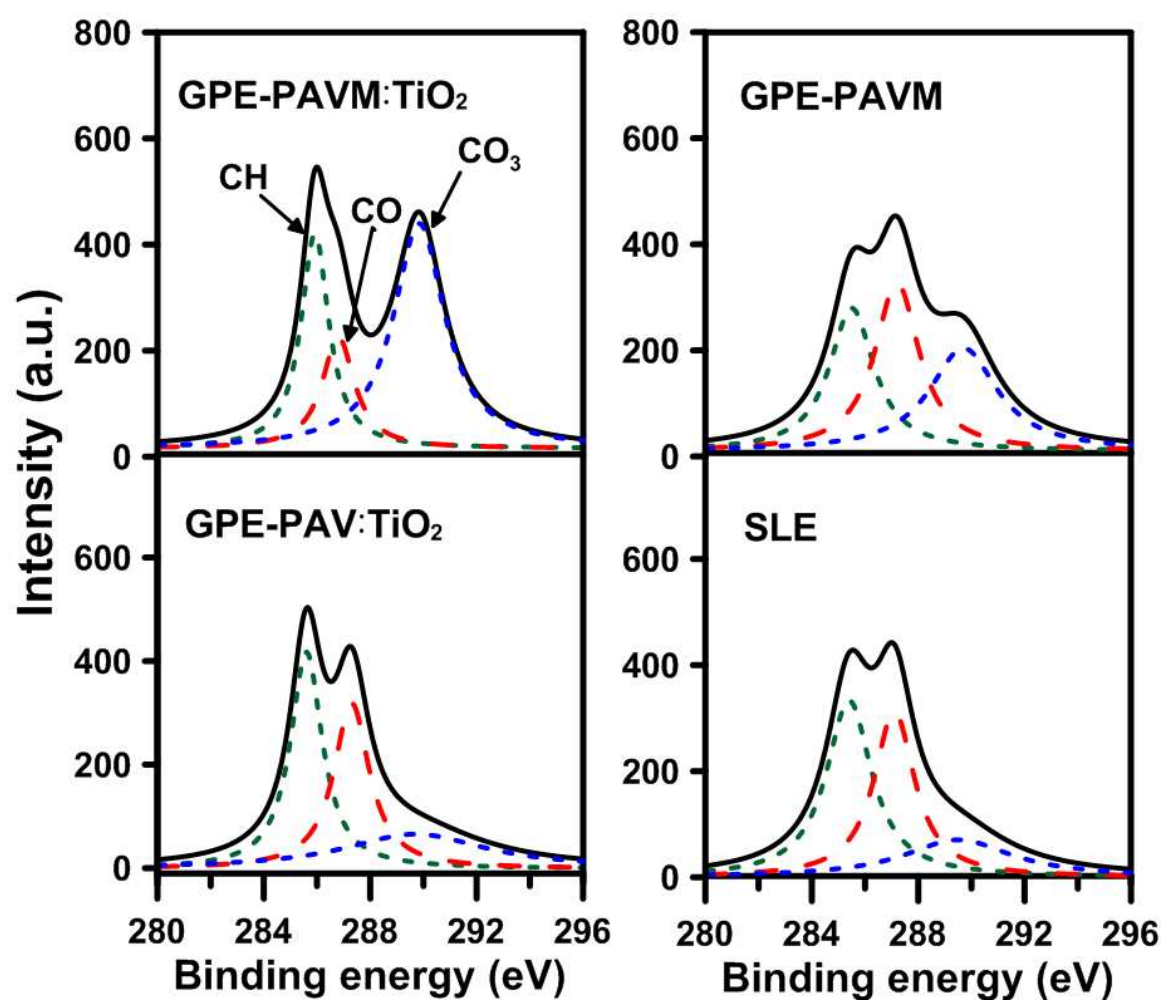

Figure S6. C 1s XPS spectra of SEI layers formed on anodes in LIBs assembled using various electrolytes and subjected to a series of charge-discharge cycles. The spectra were decomposed into $\mathrm{CH}, \mathrm{CO}$ and $\mathrm{CO}_{3}$ peaks (indicated by the dash lines), which were fitted using a Lorentzian function. ${ }^{1-3}$ 
7. Discharge capacity of graphite|electrolyte $\mid \mathrm{LiFPO}_{4}$ batteries at various C-rates

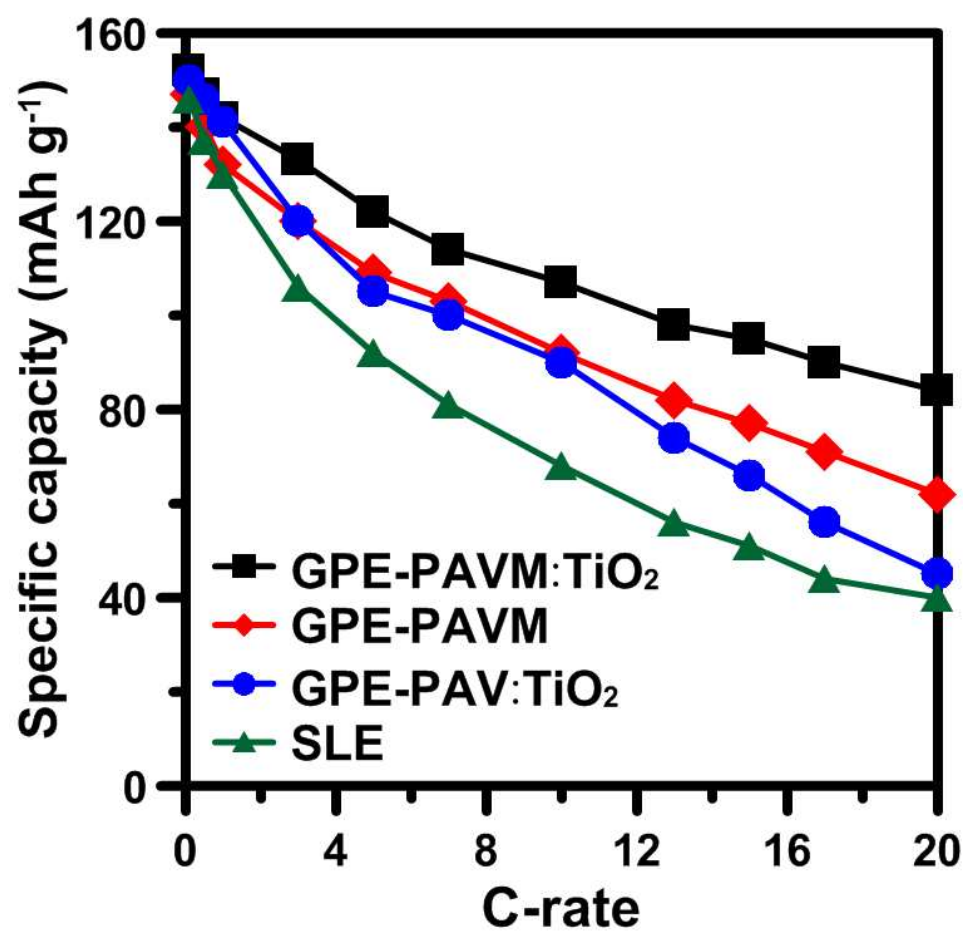

Figure S7. Discharge capacities of the graphite|electrolyte|LiFPO 4 batteries from $0.1 \mathrm{C}$ to $20 \mathrm{C}$ using various electrolytes. 


\section{References}

(1) Lee, W. J.; Prasanna, K.; Jo, Y. N.; Kim, K. J.; Kim, H. S.; Lee, C. W. Depth Profile Studies on Nickel Rich Cathode Material Surfaces after Cycling with an Electrolyte Containing Vinylene Carbonate at Elevated Temperature. Phys. Chem. Chem. Phys. 2014, 16, 17062-17071.

(2) Wang, F. M.; Cheng, H. M.; Wu, H. C.; Chu, S. Y.; Cheng, C. S.; Yang, C. R. Novel SEI Formation of Maleimide-Based Additives and its Improvement of Capability and Cyclicability in Lithium Ion Batteries. Electrochim. Acta 2009, 54, $3344-3351$.

(3) Ciosek Högström, K.; Malmgren, S.; Hahlin, M.; Rensmo, H.; Thébault, F.; Johansson, P.; Edström, K. The Influence of PMS-Additive on the Electrode/Electrolyte Interfaces in $\mathrm{LiFePO}_{4} /$ Graphite Li-Ion Batteries. J. Phys. Chem. C 2013, 117, 23476-23486. 